\title{
Mood and transient cardiac dysfunction in everyday life
}

\author{
Mimi R. Bhattacharyya $\cdot$ Andrew Steptoe
}

Received: January 5, 2010 / Accepted: July 2, 2010/Published online: July 17, 2010

(C) The Author(s) 2010. This article is published with open access at Springerlink.com

\begin{abstract}
Emotion in daily life may be associated with transient myocardial ischemia, ventricular tachycardia and impaired autonomic function in cardiac patients, but the precise temporal sequence is unclear. Eighty-eight patients with suspected coronary artery disease underwent 24-h electrocardiographic monitoring, and affect was measured with the Day Reconstruction Method. Thirteen patients $(15 \%)$ experienced one or more episodes of ST depression or ventricular tachycardia, nine of whom provided concurrent mood data. Mood and heart rate variability were analyzed for the $15 \mathrm{~min}$ before, during, and $15 \mathrm{~min}$ after each ST depression/ventricular tachycardia episode, and were compared with control periods not associated with cardiac dysfunction. Patients reported more negative mood in the 15 min preceding cardiac dysfunction compared with control periods $(P=0.02)$. Heart rate increased in the 5 min before cardiac dysfunction $(P=0.005)$, whereas low frequency heart rate variability was reduced at onset but not before cardiac dysfunction $(P=0.007)$. There were not changes in high frequency heart rate variability. This small study indicates that emotional state may contribute to vulnerability of cardiac dysfunction in everyday life.
\end{abstract}

Keywords Myocardial ischemia $\cdot$ Mood $\cdot$ Heart rate variability $\cdot$ Ventricular tachycardia $\cdot$ Coronary artery disease

Electronic supplementary material The online version of this article (doi:10.1007/s10865-010-9280-x) contains supplementary material, which is available to authorized users.

M. R. Bhattacharyya · A. Steptoe ( $\square)$

Department of Epidemiology and Public Health, University

College London, 1-19 Torrington Place, London WC1E 6BT, UK

e-mail: a.steptoe@ucl.ac.uk

\section{Introduction}

There is growing evidence that emotional factors are associated with cardiovascular health. Mood states have been related to the long term etiology of coronary heart disease (Nicholson et al. 2006) and may act as acute triggers of cardiovascular events (Bhattacharyya and Steptoe 2007; Strike and Steptoe 2005). Emotional states in everyday life may also contribute to episodes of cardiac dysfunction, including transient myocardial ischemia and ventricular tachycardia, in patients with established coronary artery disease. This association has been studied by coupling Holter monitoring with self-completed diaries in which patients rate their moods and activities (Culic et al. 2004; Gabbay et al. 1996; Krantz et al. 1996). For example, Gullette et al. (1997) found that the risk of silent transient myocardial ischemia was increased markedly in the hour following diary reports of negative emotions such as tension, sadness and frustration compared with control periods. Cardiac autonomic dysregulation may contribute to these responses, since increases in heart rate and reductions in high frequency and low frequency heart rate variability have been described in the minutes before onset of ischemia (Kop et al. 2001). It has been argued that there is a direct association between mental activity and vagal withdrawal during transient ischemia (Tuininga et al. 1995).

However, investigating the temporal sequence of mood and cardiac dysfunction presents particular methodological challenges. Diary entries may not coincide with episodes of transient myocardial ischemia or ventricular tachycardia, and compliance with diaries can be poor (Shiffman et al. 2008). In this study, we investigated mood over the monitoring period using the recently developed Day Reconstruction Method (Kahneman et al. 2004). This 
retrospective method for characterizing daily life experience combines features of time-budget measurement and experience sampling, and involves recall of the previous day as a continuous sequence of episodes, like a movie. Each episode is characterized in terms of onset and duration, location, social interaction, and activity. Participants also rate each episode on a series of affect scales. Although the Day Reconstruction Method involves recall, it is designed to increase the accuracy of emotional recollection by involving retrieval of the specific features of each episode. It has been used in a number of studies, including investigations of well-being, personality, and pain (Krueger and Stone 2008; White and Dolan 2009). We hypothesized that episodes of transient myocardial ischemia and ventricular tachycardia would be preceded by negative mood states in patients with suspected coronary artery disease, and that they would be accompanied by alterations in cardiac autonomic activity. We also assessed whether the magnitude of cardiac autonomic changes during episodes of cardiac dysfunction were associated with prevailing levels of positive and negative mood.

\section{Methods}

\section{Patients}

Participants were 88 patients recruited from Rapid Access Chest Pain clinics in three London hospitals. They had been referred by general practitioners or hospital physicians because of chest pain, and were regarded as probable coronary artery disease cases on the basis of symptomatology plus positive exercise tests or positive myocardial perfusion scans. They participated in the study prior to coronary angiography. Patients were excluded if they were prescribed antidepressant medication, could not speak English, suffered from significant non cardiac (cancer, renal, pulmonary or neurological) disease, or other cardiac disorders (heart failure, valvular disease, major arrhythmia). One hundred and forty-four patients were eligible to take part, of whom 56 refused. Patients who declined were significantly older (mean 64.9, SD 10.2 years) than those who took part (mean 61.1, SD 9.8 years, $P=0.032$ ), but did not differ in gender distribution. The study was approved by the medical research ethics committees of the participating hospitals, and all patients provided signed consent.

\section{Procedure}

Participants attended the research laboratory on the morning of day 1 between 9:00 and 12:00 h. They were equipped with a $24 \mathrm{~h}$ digital Holter monitor (Lifecard CF,
Del Mar Reynolds, Hertford, UK), as described previously (Bhattacharyya et al. 2008). The patient returned to the laboratory at the same time on the next day (day 2), at which time the Day Reconstruction Method was completed through interview with one of the investigators. Patients continued with their usual medication throughout the study.

\section{Electrocardiographic assessment}

The electrocardiographic records were analyzed by Hertford Medical (Hertford, UK) for episodes of ST segment depression and ventricular tachycardia. ST depression was defined as horizontal or downsloping ST depression $>1 \mathrm{~mm}$ or $1.5 \mathrm{~mm}$ upsloping below the isoelectric baseline, measured at $0.08 \mathrm{~s}$ after $\mathrm{J}$ point and persisting for at least $60 \mathrm{~s}$. Ventricular tachycardia was defined as three or more consecutive ventricular beats $\geq 120$ beats per minutes.

The electrocardiogram was recorded for $22-26 \mathrm{~h}$ in three channels with a six electrode array, and was digitized at $125 \mathrm{~Hz}$. Heart rate variability was assessed in the frequency domain using the HRV Tools 1.72 (Del Mar Reynolds). The $24 \mathrm{~h}$ sequence was analyzed in $30 \mathrm{~min}$ segments, each composed of up to six 5-min periods. Five minute periods with valid data were averaged to derive each 30 min segment. The following variables were computed: Total power $\left(\mathrm{ms}^{2}\right)$, very low frequency power between the limits 0.003 and $0.04 \mathrm{~Hz}\left(\mathrm{~ms}^{2}\right)$, low frequency power in the range $0.04-0.15 \mathrm{~Hz}\left(\mathrm{~ms}^{2}\right)$, and high frequency power in the range $0.15-0.40 \mathrm{~Hz}\left(\mathrm{~ms}^{2}\right)$. High frequency power is thought to be an indicator of parasympathetic tone, whereas low frequency power reflects the balance between sympathetic and vagal tone (Task Force 1996). We normalized low and high frequency power, defined as the relative power of each component in proportion to total power-very low frequency power, to account for variations in absolute power between participants (Task Force 1996). Heart rate was also analyzed.

\section{Day reconstruction method assessment}

Mood, social situations and activities over the study period were assessed using the Day Reconstruction Method, as described by Kahneman et al. (2004). Patients were asked to recall the $24 \mathrm{~h}$ monitoring period as a continuous series of episodes. They provided information about the time of onset and the duration of each episode, its physical location, social situation and activity. The minimum episode duration was $15 \mathrm{~min}$, and episodes were timed to the nearest $5 \mathrm{~min}$. After the complete $24 \mathrm{~h}$ had been reconstructed, patients rated their feelings during each episode 
on 7-point mood scales from $0=$ not at all to $6=$ very much. An aggregate summary mood measure was generated by averaging ratings of angry/hostile, depressed, and worried/anxious mood ratings and subtracting them from ratings of happiness. Higher scores indicate more positive mood. The Day Reconstruction Method appears to have good reliability (Krueger and Schkade 2008), and the measures of mood show good agreement with ecological momentary assessments (Dockray et al. in press).

\section{Other measures}

Prior history of coronary heart disease was obtained from clinical notes. Medication, socio-demographic, psychological and behavioral information was collected at interview. Medications were grouped into the following major categories: beta-blockers, statins, aspirin, angiotensin converting enzyme inhibitors, and psychotropic medication (antidepressants and anxiolytics). Anxiety was measured using the anxiety subscale from the Hospital Anxiety and Depression scale (Zigmond and Snaith 1983), and depressed mood with the Beck Depression Inventory (Beck et al. 1988).

Mood and cardiovascular correlates of ST depression/ ventricular tachycardia

Nine patients experienced one or more episodes of ST depression and four experienced ventricular tachycardia (one had both ST depression and ventricular tachycardia). Investigation of the mood and cardiovascular responses surrounding each ST depression/ventricular tachycardia episode was carried out by identifying the minute of onset and offset of each episode. The primary mood analyses were conducted on the summary mood measure, but data for individual moods were also analyzed. Five minute segments of Day Reconstruction Method mood and heart rate variability measures were then computed for the three 5 min segments preceding onset $(-15,-10$ and -5$)$, the onset segment, the segment beginning at offset of the cardiac dysfunction $(+5)$, and the two subsequent $5 \mathrm{~min}$ segments $(+10,+15)$. This procedure made it possible to identify acute changes leading up to and following each episode of cardiac dysfunction. These measures were compared with control periods involving no cardiac dysfunction. The control periods were the corresponding time points for the hour before and the hour after the ST depression/ventricular tachycardia episode; for example, if the episode of ST depression/ventricular tachycardia began at 10:05 $\mathrm{h}$ and ended at 10:17 $\mathrm{h}$, we averaged control data centered on 9:05-9:17 $\mathrm{h}$ and 11:05-11:17 h. When patients had more than one episode of ST depression/ventricular tachycardia, results were averaged.
Statistical analysis

Patients with and without ST depression/ventricular tachycardia period were compared with analysis of variance for continuous and $\chi^{2}$ analyses for nonparametric measures. Some values were missing, so the percentages presented are adjusted accordingly, and denominators are included in the description of results. The average control mood and heart rate variability were compared with the ST depression/ventricular tachycardia period using repeated measures analysis of variance, with period (ischemia or control) and segment (the 7 within-period segments) as within-person factors. The Greenhouse Geisser correction was applied when assumptions of sphericity were violated. Post hoc tests were carried out using Tukey's least significant difference test. Additional analyses involved the use of beta-blockers and psychotropic medication as between-person factors. The relationship between prior mood and cardiac responses during episodes of cardiac dysfunction was assessed using product-moment correlations.

\section{Results}

Three patients had a single episode of ST depression, two patients had two episodes, and four patients had three episodes. These episodes lasted from 1 to $17 \mathrm{~min}$, and occurred at a range of times of day and night, with a high proportion on the morning of day 2 (13/19 episodes). The ventricular tachycardia episodes were mostly short, although one lasted more than $10 \mathrm{~min}$. These episodes of cardiac dysregulation were predominantly silent. Analyses of heart rate variability were carried out with all participants. The Day Reconstruction Method analyses were only possible with 9 of the 13 patients (6 ST depression/3 ventricular tachycardia) because Day Reconstruction Method data were lost for one patient, and the episodes of cardiac dysregulation occurred during sleep for the remaining three.

The group of patients who experienced ST depression/ ventricular tachycardia included 8 men and 5 women, with an average age of $64.3 \pm 9.4$ years. The demographic, clinical and psychological characteristics of patients with and without ST depression/ventricular tachycardia are summarized in Table 1. There were no differences between the patients who showed cardiac dysregulation and the remainder in age, gender or ethnic distribution, educational attainment, marital status, body mass index, diabetes, hypertension, or in the use of statins, and aspirin. Although not significant, it is notable that there were fewer smokers in the ST depression/ventricular tachycardia group (7.7 vs. $26.7 \%$ ), and also fewer patients taking angiotensin converting enzyme inhibitors (15.4 vs. $38.8 \%$ ). Additionally, 
Table 1 Characteristics of patients with ST depression/Ventricular tachycardia Means (standard deviation) and N/Denominator (percentage)

\begin{tabular}{llcl}
\hline & Normal ECG & $\begin{array}{l}\text { ST depression/ventricular } \\
\text { tachycardia }\end{array}$ & Differences $(P)$ \\
\hline Gender $($ men $)$ & $52 / 75(69.3 \%)$ & $8 / 13(61.5 \%)$ & 0.75 \\
Age (years) $n=88)$ & $61.20(9.7)$ & $63.85(8.8)$ & 0.36 \\
Ethnicity (white) & $55 / 75(73.3 \%)$ & $8 / 13(61.5 \%)$ & 0.51 \\
Education (secondary) & $39 / 75(52.0 \%)$ & $8 / 13(61.5 \%)$ & 0.56 \\
Marital status (married) & $45 / 75(60.0 \%)$ & $8 / 13(61.5 \%)$ & 1.00 \\
Current smoker & $20 / 75(26.7 \%)$ & $1 / 13(7.7 \%)$ & 0.18 \\
Body mass index $\left(\mathrm{kg} / \mathrm{m}^{2}\right)(n=87)$ & $28.23(5.7)$ & $26.35(3.6)$ & 0.12 \\
Diabetes & $11 / 74(14.9 \%)$ & $3 / 11(27.3 \%)$ & 0.38 \\
Hypertension & $41 / 75(54.7 \%)$ & $6 / 13(46.2 \%)$ & 0.76 \\
Beta-blocker medication & $54 / 75(72.0 \%)$ & $5 / 13(38.5 \%)$ & 0.026 \\
Statin medication & $63 / 75(84.0 \%)$ & $9 / 13(69.2 \%)$ & 0.24 \\
Aspirin medication & $65 / 74(87.8 \%)$ & $12 / 13(92.3 \%)$ & 0.99 \\
Angiotensin converting enzyme & $26 / 67(38.8 \%)$ & $2 / 13(15.4 \%)$ & 0.13 \\
$\quad$ inhibitor medication & & & \\
Psychotropic medication & $3 / 75(4.0 \%)$ & $3 / 13(23.1 \%)$ & 0.039 \\
Anxiety score $(n=88)$ & $6.41(3.8)$ & $7.31(3.4)$ & $0.42(4.9)$ \\
Depression score $(n=88)$ & $10.16(7.5)$ & $6 / 13(46.2 \%)$ & 0.93 \\
Definite coronary artery disease & $50 / 75(66.7 \%)$ & 0.21 \\
\hline
\end{tabular}

the patients showing ST depression/ventricular tachycardia were less likely to be taking beta-blocker medication than the remainder (38.5 vs. $72.0 \%, P=0.03$ ), and were more likely to be taking psychotropic medication (23.1 vs. $4.0 \%$, $P=0.039$ ). Anxiety averaged $6.55 \pm 3.7$ and depression $10.2 \pm 7.1$; these values did not differ between patients with and without ST depression/ventricular tachycardia. Nor was there any difference in the proportion of patients with definite coronary artery disease as detected on angiography, though it appeared that slightly fewer patients who displayed cardiac dysregulation during the study period had definite coronary artery disease (46.2 vs. $66.7 \%$ ). The characteristics of the nine patients in whom acute mood responses were analyzed were the same as those of all 13 with ST depression/ventricular tachycardia.

Patients reported an average of 17.3 episodes over the day using the Day Reconstruction Method, with a range of 13-20. The average duration of episodes was $60.2 \mathrm{~min}$ (range 43.6-88.9 across individuals). These findings did not differ between patients with and without ST depression/ventricular tachycardia.

Mood and cardiac dysfunction

The analysis of aggregated mood showed a significant period by segment interaction, $F(6,48)=8.68$, $P=0.008, \eta^{2}=0.52$, illustrated in Fig. 1. Separate analyses of the ST depression/ventricular tachycardia periods were carried out to determine which in period changes over the 5 min episodes occurred. These confirmed significant changes over segments of the cardiac dysfunction, $F$ (6, $48)=5.47, P=0.048$, but not control periods, $F(6$, $48)=1.32, P=0.27$ ). Mood was more negative in the 15 min preceding cardiac dysfunction than in control periods; following onset of cardiac dysfunction, mood became more positive $(P<0.05)$. In analyses of the separate mood ratings, significant effects were present for happiness, $F(6,48)=6.24, P=0.037 \eta^{2}=0.044$, and worry, $F(6,48)=4.44, P=0.006, \eta^{2}=0.31$, that mirrored the aggregate responses, but not for depressed mood, anger or tiredness. Since the sample size is small, Table 2 details the medians and interquartile ranges for these data.

Heart rate variability and cardiac dysfunction

There were significant period by segment interactions for heart rate $\left(P=0.008, \quad \eta^{2}=0.78\right)$ and LF-HRV $\left(P=0.007, \eta^{2}=0.46\right)$. Post hoc tests indicated that heart rate did not change in the control period, but increased in the 5 min before onset of cardiac dysfunction, reaching its peak immediately after episode (Fig. 1). The increase averaged 16.8 beats per minute (see Table 2). By contrast, low frequency heart rate variability decreased at the onset of cardiac dysfunction, remaining low until the episode terminated (Fig. 1). There were no significant changes in very low or high frequency heart rate variability associated with cardiac dysfunction. The high/low frequency ratio showed a near-significant period by segment interaction 

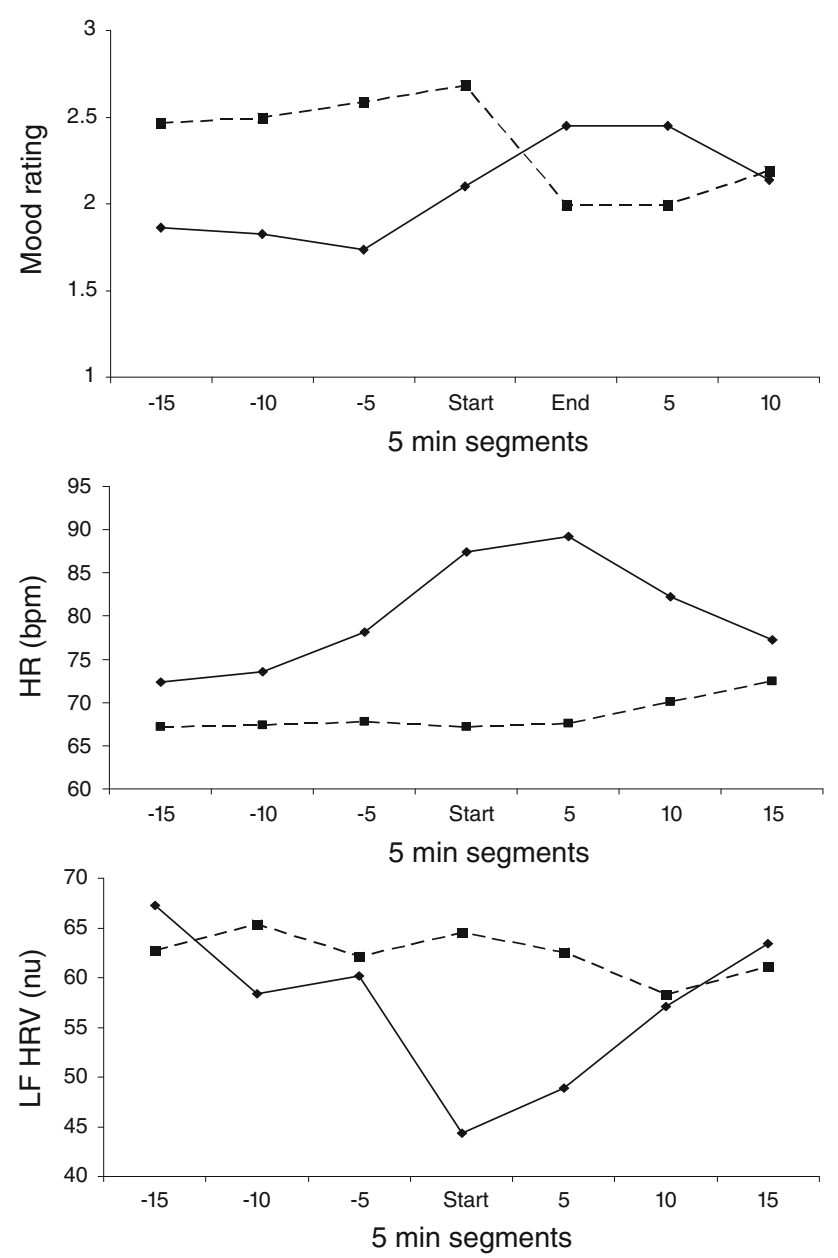

Fig. 1 Mean levels of overall mood (upper panel), heart rate (middle panel) and low frequency heart rate variability (lower panel) in the period surrounding episodes of ST depression/ventricular tachycardia (solid lines-diamond) or control periods (dashed line-squares). Averages for the three $5 \mathrm{~min}$ periods before each episode $(-15,-10$, -5 ), the $5 \mathrm{~min}$ from the start of the episode (Start), and the three 5 min periods starting at the end of each episode $(5,10,15)$ are plotted

$\left(P=0.052, \eta^{2}=0.28\right)$, but in post hoc tests none of the segments differed between cardiac dysfunction and control periods. Figures detailing the responses of individual patients are available as electronic supplementary material.

Similar profiles of mood and cardiac response were observed when analyses were limited either to episodes of ST depression or ventricular tachycardia alone. We also assessed whether effects differed in patients who did and did not take beta-blockers, and those who were or were not taking psychotropic medication. The period by segment interactions remained significant for heart rate after betablockers and psychotropic medication had been included as between-person factors ( $P=0.006$ and 0.003 , respectively). There was no main effect for use of either medication, and no interaction between medication use and phase or segment.
Similarly, the period by segment interaction for low frequency heart rate variability remained significant after including medication as a between-person factor $(P=0.009$ in both cases), and there were no interactions with phase or segment. So the pattern of responses associated with cardiac dysfunction was unaffected by medication use.

\section{Discussion}

This small study assessed the association between mood in everyday life and autonomic function during episodes of ST depression or ventricular tachycardia in patients with suspected coronary artery disease, using the Day Reconstruction Method to monitor mood. Episodes of ST depression or ventricular tachycardia were experienced by $14.8 \%$ of patients during the $24 \mathrm{~h}$ of electrocardiographic monitoring. Cardiac dysfunction was preceded by negative moods for at least $15 \mathrm{~min}$, whereas autonomic changes were more immediate. Heart rate increased in the $5 \mathrm{~min}$ before onset, followed by reductions in low frequency heart rate variability coinciding with ST depression/ventricular tachycardia. Although it cannot be concluded that more negative moods stimulated autonomic changes, the temporal sequence is consistent with this view.

The incidence of silent transient myocardial ischemia during ambulatory electrocardiographic monitoring is highly variable across studies (Xanthos et al. 2008). For example, Gullette et al. (1997) reported that $44 \%$ of patients who had been withdrawn from beta-blockers and other medications manifest transient ischemia over $48 \mathrm{~h}$, whereas the rate in a more recent study of hypertensive patients was $20.1 \%$ (Uen et al. 2006). Most of the patients in this study were taking beta-blockers, and the incidence of ST depression/ventricular tachycardia was greater among those who were not taking beta-blockers (Table 1). However, analyses of heart rate and heart rate variability responses over the periods of cardiac dysfunction did not uncover any differences between those who did and did not use beta-blockers. Consequently, the presence or absence of beta-blockade is unlikely to be responsible for the pattern of response detailed in Fig. 1, although it probably did reduce the overall incidence of cardiac dysfunction. By the same token, the pattern of responses was comparable in patients who were or were not taking psychotropic medication.

It is interesting that ST depression/ventricular tachycardia was no more common among patients who were subsequently found to have significant coronary artery obstruction than those who were not classified as having coronary artery disease. The definition of significant coronary artery disease is somewhat arbitrary, and depends on the identification of major stenosis. Diffuse atheroma or 
Table 2 Mean, standard deviation, median and interquartile ranges for mood, heart rate, and low frequency heart rate variability

\begin{tabular}{|c|c|c|c|c|c|c|c|c|}
\hline & \multicolumn{4}{|c|}{ ST Depression/ventricular tachycardia period } & \multicolumn{4}{|c|}{ Control period } \\
\hline & Mean & SD & Median & $\begin{array}{l}\text { Interquartile } \\
\text { range }\end{array}$ & Mean & SD & Median & $\begin{array}{l}\text { Interquartile } \\
\text { range }\end{array}$ \\
\hline \multicolumn{9}{|c|}{ Overall mood } \\
\hline$-15 \min$ & 1.86 & 3.47 & 1.78 & 4.17 & 2.47 & 2.64 & 3.00 & 3.85 \\
\hline$-10 \min$ & 1.83 & 3.56 & 1.78 & 4.17 & 2.50 & 2.65 & 3.11 & 3.85 \\
\hline$-5 \min$ & 1.73 & 3.54 & 1.77 & 4.42 & 2.59 & 2.71 & 3.11 & 3.89 \\
\hline Start & 2.10 & 3.62 & 2.89 & 4.42 & 2.69 & 2.79 & 3.50 & 4.22 \\
\hline $5 \mathrm{~min}$ & 2.45 & 3.33 & 2.89 & 3.92 & 2.00 & 3.30 & 2.75 & 4.31 \\
\hline $10 \mathrm{~min}$ & 2.45 & 3.32 & 2.88 & 3.92 & 2.00 & 3.30 & 2.75 & 4.31 \\
\hline $15 \mathrm{~min}$ & 2.14 & 3.23 & 3.00 & 4.39 & 2.19 & 3.33 & 3.00 & 4.00 \\
\hline \multicolumn{9}{|c|}{ Heart rate (bpm) } \\
\hline$-15 \min$ & 72.4 & 16.7 & 74.8 & 21.1 & 67.2 & 14.7 & 64.5 & 20.8 \\
\hline$-10 \min$ & 73.6 & 18.0 & 75.1 & 25.5 & 67.4 & 13.9 & 65.3 & 20.4 \\
\hline$-5 \min$ & 78.1 & 18.0 & 78.7 & 25.2 & 67.8 & 12.8 & 68.3 & 18.3 \\
\hline Start & 87.5 & 24.8 & 88.8 & 46.2 & 67.2 & 12.2 & 67.5 & 21.1 \\
\hline $5 \mathrm{~min}$ & 89.2 & 22.5 & 89.2 & 42.5 & 67.6 & 11.5 & 67.9 & 21.0 \\
\hline $10 \mathrm{~min}$ & 82.2 & 19.4 & 80.4 & 25.7 & 70.1 & 14.1 & 67.2 & 24.3 \\
\hline $15 \mathrm{~min}$ & 77.3 & 12.7 & 79.1 & 11.1 & 72.5 & 15.2 & 74.0 & 30.0 \\
\hline \multicolumn{9}{|c|}{$L F H R V(n u)$} \\
\hline$-15 \min$ & 67.3 & 20.5 & 69.9 & 23.6 & 62.7 & 12.6 & 64.9 & 20.0 \\
\hline$-10 \mathrm{~min}$ & 58.4 & 23.6 & 57.6 & 47.5 & 65.4 & 19.0 & 66.4 & 25.2 \\
\hline$-5 \min$ & 60.2 & 21.5 & 67.6 & 40.5 & 62.1 & 17.3 & 67.8 & 20.0 \\
\hline Start & 44.4 & 21.0 & 41.5 & 36.2 & 64.5 & 14.6 & 61.9 & 24.3 \\
\hline $5 \mathrm{~min}$ & 48.9 & 27.5 & 52.4 & 41.1 & 62.6 & 13.5 & 65.6 & 19.9 \\
\hline $10 \mathrm{~min}$ & 57.1 & 28.2 & 66.3 & 39.7 & 58.3 & 22.4 & 55.2 & 35.3 \\
\hline $15 \mathrm{~min}$ & 63.4 & 20.2 & 69.0 & 27.9 & 61.1 & 20.3 & 68.0 & 28.0 \\
\hline
\end{tabular}

microvascular coronary dysfunction may be present in these individuals (Phan et al. 2009).

The pattern of autonomic response was broadly consistent with previous studies in which transient myocardial ischemia has been preceded by vagal withdrawal and/or sympathetic activation (Tuininga et al. 1995; Uen et al. 2006). Kop et al. (2001) reported a reduction of low frequency heart rate variability within the 5 min preceding ST depression, whereas heart rate increased several minutes earlier. We also showed that heart rate increases occurred before alterations in low frequency heart rate variability. In an analysis of 8 cases of ischemic sudden death during ambulatory electrocardiographic monitoring, Pozzati et al. (1996) found that all showed ST segment shifts and 5 of the 8 had ventricular tachyarrhythmias which were preceded by marked reductions in heart rate variability. However, it is notable that high frequency heart rate variability did not show a conspicuous response pattern related to transient cardiac dysfunction. The small sample size in this study may have prevented such an effect from emerging. It is also possible that the lack of high frequency response was due to the assessment of patients with suspected coronary artery disease rather than established coronary heart disease, or because the temporal resolution was 5 min rather than a shorter interval. However, the absence of differences in high frequency coupled with the low frequency heart rate variability responses suggests that effects on sympathetic activation are more prominent than those on vagal withdrawal.

This study used normalized rather than absolute power in the high and low frequency wavebands to represent heart rate variability. Normalization of heart rate variability is a means of reducing the impact of differences in total power, and this is particularly relevant in a small study of this type. Additionally, normalization emphasizes the controlled balance between the two branches of the autonomic nervous system. Although the Task Force (1996) on the assessment of heart rate variability suggested that absolute power is presented along with normalized units, these data are not included in the interests of space.

We used the Day Reconstruction Method to assess moods in the periods surrounding each episode. This method has the advantage that it does not interfere with ongoing activities, as in the case of concurrent diary 
measures, and provides a fine grained analysis of changes over time. The technique has previously been used to investigate mood changes over the day (Stone et al. 2006) and the experience of pain in every day life (Krueger and Stone 2008), and has good reliability and comparability with momentary assessments of mood (Dockray et al. in press; Krueger and Schkade 2008). A limitation of the method in the present context lies in its temporal resolution and ability to identify very brief affective episodes. We set $15 \mathrm{~min}$ to be the minimum length of episode permitted, and measured episodes to the nearest $5 \mathrm{~min}$. This has been commonly adopted in Day Reconstruction Method studies for two reasons: first, to help people understand that what is meant by an episode is not a very brief occurrence, but an activity or interaction of substance. Second, division of the day into very short episodes of a few minutes makes the method unwieldy. However, this means that the method was not able to identify mood fluctuations shorter than $5 \mathrm{~min}$, and very brief but potentially significant changes in affective state could not be measured.

Mood was more negative in the $15 \mathrm{~min}$ before onset of cardiac dysfunction than in comparison control periods, or the minutes after onset. This pattern was driven by reductions in positive mood and increases in worry. Gullette et al. (1997) have previously shown with diary assessments that cardiac dysfunction is preceded by increased ratings of tension, sadness and frustration. Interestingly, the autonomic changes indicative of vagal withdrawal were greater among people with more negative moods. Bacon et al. (2004) reported that negative emotional states were associated with decreased low and high frequency heart rate variability, and positive emotions with increased low frequency activity, independently of medication and other factors, whereas Kop et al. (2001) showed that ambulatory ischemic episodes that occurred during high mental activity were accompanied by more pronounced heart rate increases and reductions in low frequency heart rate variability. The findings are consistent with the hypothesis that emotional states may contribute to the autonomic dysregulation that is associated with transient cardiac dysfunctions in patients with coronary artery disease.

The number of individuals experiencing ST depression or ventricular tachycardia was small, so there were insufficient cases to analyze ST depression and ventricular tachycardia episodes separately. We did not monitor physical activity in this study, and it is possible that the cardiac dysfunction and control periods differed in activity levels. However, it is striking that the mean heart rate was very similar at the beginning and end of cardiac dysfunction and control periods, suggesting that sustained differences in concurrent physical activity were not present. A longer period of ambulatory monitoring would have been advantageous, and other studies of emotional and physical triggers have involved $48 \mathrm{~h}$ rather than $24 \mathrm{~h}$ measurement (Gabbay et al. 1996; Gullette et al. 1997; Kop et al. 2001). It cannot of course be concluded that more negative moods are typically followed by cardiac dysfunction, but rather that negative emotional states may precede the cardiac autonomic changes involved in cardiac dysfunction in everyday life. The Day Reconstruction Method may prove a valuable tool in the investigation of mood changes associated with cardiac dysfunction in patients with coronary artery disease.

Acknowledgments This research was supported by the British Heart Foundation (RG/05/006). The funding source has not been involved in the submission of the manuscript or in the decision to publish the data.

Open Access This article is distributed under the terms of the Creative Commons Attribution Noncommercial License which permits any noncommercial use, distribution, and reproduction in any medium, provided the original author(s) and source are credited.

\section{References}

Bacon, S. L., Watkins, L. L., Babyak, M., Sherwood, A., Hayano, J., Hinderliter, A. L., et al. (2004). Effects of daily stress on autonomic cardiac control in patients with coronary artery disease. American Journal of Cardiology, 93, 1292-1294.

Beck, A. T., Steer, R. A., \& Garbin, M. G. (1988). Psychometric properties of the Beck depression inventory: Twenty-five years of evaluation. Clinical Psychology Review, 8, 77-100.

Bhattacharyya, M. R., \& Steptoe, A. (2007). Emotional triggers of acute coronary syndromes: Strength of evidence, biological processes, and clinical implications. Progress in Cardiovascular Disease, 49, 353-365.

Bhattacharyya, M. R., Whitehead, D. L., Rakhit, R., \& Steptoe, A. (2008). Depressed mood, positive affect, and heart rate variability in patients with suspected coronary artery disease. Psychosomatic Medicine, 70, 1020-1027.

Culic, V., Eterovic, D., Miric, D., Giunio, L., Lukin, A., \& Fabijanic, D. (2004). Triggering of ventricular tachycardia by meteorologic and emotional stress: Protective effect of beta-blockers and anxiolytics in men and elderly. American Journal of Epidemiology, 160, 1047-1058.

Dockray, S., Grant, N., Stone, A. A., Kahneman, D., Wardle, J., \& Steptoe, A. (in press). A comparison of affect ratings obtained with ecological momentary assessment and the day reconstruction method. Social Indicators Research.

Force, Task. (1996). Heart rate variability: Standards of measurement, physiological interpretation and clinical use. Task Force of the European Society of Cardiology and the North American Society of Pacing and Electrophysiology. Circulation, 93, 1043-1065.

Gabbay, F. H., Krantz, D. S., Kop, W. J., Hedges, S. M., Klein, J., Gottdiener, J. S., et al. (1996). Triggers of myocardial ischemia during daily life in patients with coronary artery disease: Physical and mental activities, anger and smoking. Journal of the American College of Cardiology, 27, 585-592.

Gullette, E. C., Blumenthal, J. A., Babyak, M., Jiang, W., Waugh, R. A., Frid, D. J., et al. (1997). Effects of mental stress on myocardial ischemia during daily life. Journal of the American Medical Association, 277, 1521-1526. 
Kahneman, D., Krueger, A. B., Schkade, D. A., Schwarz, N., \& Stone, A. A. (2004). A survey method for characterizing daily life experience: The day reconstruction method. Science, 306, 1776-1780.

Kop, W. J., Verdino, R. J., Gottdiener, J. S., O'Leary, S. T., Bairey Merz, C. N., \& Krantz, D. S. (2001). Changes in heart rate and heart rate variability before ambulatory ischemic events. Journal of the American College of Cardiology, 38, 742-749.

Krantz, D. S., Kop, W. J., Gabbay, F. H., Rozanski, A., Barnard, M., Klein, J., et al. (1996). Circadian variation of ambulatory myocardial ischemia. Triggering by daily activities and evidence for an endogenous circadian component. Circulation, 93, 13641371.

Krueger, A., \& Schkade, D. (2008). The reliability of subjective wellbeing measures. Journal of Public Economics, 92, 1833-1845.

Krueger, A. B., \& Stone, A. A. (2008). Assessment of pain: A community-based diary survey in the USA. Lancet, 371, 15191525.

Nicholson, A., Kuper, H., \& Hemingway, H. (2006). Depression as an aetiologic and prognostic factor in coronary heart disease: A meta-analysis of 6362 events among 146538 participants in 54 observational studies. European Heart Journal, 27, 2763-2774.

Phan, A., Shufelt, C., \& Merz, C. N. (2009). Persistent chest pain and no obstructive coronary artery disease. Journal of the American Medical Association, 301, 1468-1474.

Pozzati, A., Pancaldi, L. G., Di Pasquale, G., Pinelli, G., \& Bugiardini, R. (1996). Transient sympathovagal imbalance triggers "ischemic" sudden death in patients undergoing electrocardiographic Holter monitoring. Journal of the American College of Cardiology, 27, 847-852.
Shiffman, S., Stone, A. A., \& Hufford, M. R. (2008). Ecological momentary assessment. Annual Review of Clinical Psychology, $4,1-32$.

Stone, A. A., Schwartz, J. E., Schkade, D., Schwarz, N., Krueger, A., \& Kahneman, D. (2006). A population approach to the study of emotion: Diurnal rhythms of a working day examined with the Day Reconstruction Method. Emotion, 6, 139-149.

Strike, P. C., \& Steptoe, A. (2005). Behavioral and emotional triggers of acute coronary syndromes: A systematic review and critique. Psychosomatic Medicine, 67, 179-186.

Tuininga, Y. S., Crijns, H. J., Brouwer, J., van den Berg, M. P., Man in't Veld, A. J., Mulder, G., et al. (1995). Evaluation of importance of central effects of atenolol and metoprolol measured by heart rate variability during mental performance tasks, physical exercise, and daily life in stable postinfarct patients. Circulation, 92, 3415-3423.

Uen, S., Un, I., Fimmers, R., Vetter, H., \& Mengden, T. (2006). Myocardial ischemia during everyday life in patients with arterial hypertension: Prevalence, risk factors, triggering mechanism and circadian variability. Blood Pressure Monitoring, 11, $173-182$.

White, M. P., \& Dolan, P. (2009). Accounting for the richness of daily activities. Psychological Science, 20, 1000-1008.

Xanthos, T., Ekmektzoglou, K. A., \& Papadimitriou, L. (2008). Reviewing myocardial silent ischemia: Specific patient subgroups. International Journal of Cardiology, 124, 139-148.

Zigmond, S., \& Snaith, R. B. (1983). The hospital anxiety and depression scale. Acta Psychiatrica, 86, 1-7. 